\title{
The proof of Riemann Hypothesis
}

\author{
Jyotirmoy Biswas \\ 7,Sreepur Lane, P.O-Haltu,KOLKATA-700078
}

The condition for which $\left(\sum_{n=1}^{\infty} \frac{1}{(2 n-1)^{Z}}\right)^{2}+\left(\sum_{n=1}^{\infty} \frac{1}{(2 n)^{Z}}\right)^{2}=0$ where $Z$ is a complex number reveals those points $Z$ for which the functions $\left(\sum_{n=1}^{\infty} \frac{1}{(2 n-1)^{Z}}\right)+i\left(\sum_{n=1}^{\infty} \frac{1}{(2 n)^{Z}}\right)$ and $\left(\sum_{n=1}^{\infty} \frac{1}{(2 n-1)^{Z}}\right)-i\left(\sum_{n=1}^{\infty} \frac{1}{(2 n)^{Z}}\right)$ have zeroes.Finally,by direct analysis we can find zeroes of Riemann zeta funtion .

\section{Introduction}

1.1 Riemann hypothesis

Theorem 1 (Riemann hypothesis) All non-trival zeroes of Riemann zeta function defined by $\zeta(Z)=\sum_{n=1}^{\infty} \frac{1}{n^{Z}}$ where $Z$ is a complex number , lie on the line $Z=\left(\frac{1}{2}+i y\right)$.

1.21

Since $\left(\sum_{n=1}^{\infty} \frac{1}{(2 n)^{Z}}\right)=\frac{1}{2^{Z}}\left[\left(\sum_{n=1}^{\infty} \frac{1}{(2 n-1)^{Z}}\right)+\left(\sum_{n=1}^{\infty} \frac{1}{(2 n)^{Z}}\right)\right]$, Therefore,

$$
\left(2^{Z}-1\right)\left(\sum_{n=1}^{\infty} \frac{1}{(2 n)^{Z}}\right)=\left(\sum_{n=1}^{\infty} \frac{1}{(2 n-1)^{Z}}\right)
$$

Consequently,

$$
\left(\sum_{n=1}^{\infty} \frac{1}{(2 n-1)^{Z}}\right)^{2}+\left(\sum_{n=1}^{\infty} \frac{1}{(2 n)^{Z}}\right)^{2}=0
$$

Implies, $\left[\left(2^{Z}\right)^{2}-(2)\left(2^{Z}\right)+2\right]\left(\sum_{n=1}^{\infty} \frac{1}{(2 n)^{Z}}\right)^{2}=0 \quad$ Implies, either $\quad\left(\sum_{n=1}^{\infty} \frac{1}{(2 n)^{Z}}\right)=0 \quad$ or $\left[\left(2^{Z}\right)^{2}-(2)\left(2^{Z}\right)+2\right]=0$ or Both. Let us assume first $\left(\sum_{n=1}^{\infty} \frac{1}{(2 n)^{Z}}\right) \neq 0$ Then, $2^{Z}=1 \pm i$ Implies, $2^{Z}=\sqrt{2}(e)^{\frac{(8 k \pm 1)(\pi)(i)}{4}}$, Where, $k$, is any positive integer including zero. Implies, $Z$ lie on the line $Z=\frac{1}{2}+\left[\frac{(8 k \pm 1)(\pi)(i)}{(4 \ln 2)}\right]$ When, any point $Z_{1}$ liesonthe line $Z=\frac{1}{2}+\left[\frac{(8 k+1)(\pi)(i)}{(4 \ln 2)}\right]$ It follows from eq (1.2.1.1),

$$
\begin{aligned}
& \left.2^{(} z_{1}\right)=1+i \\
& \left(\sum_{n=1}^{\infty} \frac{1}{\left.(2 n-1)^{(} z_{1}\right)}\right)-(i)\left(\sum_{n=1}^{\infty} \frac{1}{\left.(2 n)^{(} z_{1}\right)}\right)=0
\end{aligned}
$$

Similarly,If any point $Z_{2}$ lies on the line $Z=\frac{1}{2}+\left[\frac{(8 k-1)(\pi)(i)}{(4 \ln 2)}\right]$ Then, it follows from eq (1.2.1.1), 
Consequently,

$$
2\left(z_{2}\right)=1-i
$$

$$
\left(\sum_{n=1}^{\infty} \frac{1}{\left.(2 n-1)^{(} z_{2}\right)}\right)+i\left(\sum_{n=1}^{\infty} \frac{1}{\left.(2 n)^{(} z_{2}\right)}\right)=0
$$

But,We want something more. We wish to show that

\subsection{2}

If any point

lies on the line

$$
z_{1}
$$

$$
Z=\left(\frac{1}{2}+\left[\frac{(8 k+1)(\pi)(i)}{(4 \ln 2)}\right]\right)
$$

and another point

$$
z_{2}
$$

lies on the line

$$
Z=\left(\frac{1}{2}+\left[\frac{(8 k-1)(\pi)(i)}{(4 \ln 2)}\right]\right)
$$

Then,modulas of $\left|\sum_{n=1}^{\infty} \frac{1}{\left.(2 n-1)^{(} z_{1}\right)}\right|=\left|\sum_{n=1}^{\infty} \frac{1}{\left.(2 n-1)^{(} z_{2}\right)}\right|=\sigma$, where $\sigma$ tends to zero.

But before showing this, let us show that four common values of all infinitely many valued function

$\left.(e)^{[} \frac{(i)(\theta)(\ln p)}{\ln 2}\right]$ are $(-1), 1$ and $\pm i$ where $P$ is any odd prime taken arbitrarily.

Since

$$
\begin{aligned}
& \frac{1}{(e)^{t}-1}+\frac{1}{2}= \\
& \frac{1}{2}\left(\frac{(e)^{t}+1}{(e)^{t}-1}\right) \\
& =\frac{(i)}{2} \cot \left(\frac{1}{2} i t\right) \\
& =\frac{1}{t}+(2 t) \sum_{n=1}^{\infty} \frac{1}{t^{2}+4 n^{2}(\pi)^{2}}
\end{aligned}
$$

If $t=2(\pi)(k)(i)+\frac{(\ln p)(i)(\theta)}{\ln 2}$

where $k$ is any positive integerand $k \in(0,1,2, \ldots)$,

then as $k$ tends to $\infty$,it follows, $\frac{1}{\left.(e)^{[} 2(\pi)(k) i+\frac{(\ln p)(i)(\theta)}{\ln 2}\right]-1}+\frac{1}{2}=0$ Consequently,

$$
\left.(e)^{[} 2(\pi)(k)(i)+\frac{(\ln p)(i)(\theta)}{\ln 2}\right]=-1
$$

Since, $(e)^{2(\pi)(k) i}=1$ So it follows from eqution 1.3.2.1 that one of the values of

$$
\text { (e) } \frac{(i)(\theta)(\ln p)}{\ln 2}=-1
$$

Obviously then,chooseing suitable $\theta$ we can arrive at the values $\pm i$ and 1 . Since the matter plays a key role in what follows, an example will not be out of place here.Let us consider the many valued function 
$\underline{(\ln 3)(\pi)(i)}$

(e) $4 \ln 2$. Taking the value of $(\ln 3)$ and $(\ln 2)$ upto 9 decimal and using De Moivre's Theorem we find

$$
\begin{aligned}
& \underline{(\ln 3)(\pi)(i)} \\
& \text { (e) } 4 \ln 2= \\
& \underline{(1.098612289)(\pi)(i)} \\
& \text { (e) }(4)(0.69314718)= \\
& \text { (e) } \frac{(1.584962502)(\pi)(i)}{4}= \\
& (2-0.415037498)(\pi)(i) \\
& \text { (e) } 4= \\
& (i)(e) \frac{(103759374)(\pi)(-i)}{1000000000}= \\
& \text { (i)(e) } \quad 1000000000
\end{aligned}
$$

Obviously then,when $n=448120313$,

$$
\text { (e) } \frac{(\ln 3)(\pi)(i)}{4 \ln 2}=(-i)
$$

When $n=198120313$, then

$$
\text { (e) } \frac{(\ln 3)(\pi)(i)}{4 \ln 2}=1
$$

when $n=948120313$,then

$$
\text { (e) } \frac{(\ln 3)(\pi)(i)}{4 \ln 2}=i
$$

when $n=698120313$,then

$$
\text { (e) } \frac{(\ln 3)(\pi)(i)}{4 \ln 2}=-1
$$

Actually,among infinitely many values, these four values are common to all $(e)^{\frac{(\ln 2}{4}}$, where $p$ is any odd prime taken arbitrarily. Now since,

$$
\left(\frac{1}{1-\frac{1}{p^{z}}}\right)=\frac{p^{z}}{P^{z}-1}
$$

So,the function $\sum_{n=1}^{\infty} \frac{1}{(2 n-1)^{Z}}$ may be regarded as the rational function of two functions $\psi(z)$ and $\phi(z)$ i.e

$$
\sum_{n=1}^{\infty} \frac{1}{(2 n-1)^{Z}}=\frac{\psi(z)}{\phi(z)}
$$

where, $z=x+i y, p_{n}$ denotes the $n$th prime and 


$$
\left.\begin{array}{l}
\psi(\mathrm{z})= \\
\left.{ }_{\mathbf{n}=2}^{\Phi} \mathrm{P}^{\mathrm{X}}(\mathrm{e})^{(\ln \mathrm{p} \mathbf{n}}\right)(\mathrm{y})(\mathrm{i})
\end{array}\right]
$$

and

$$
\begin{gathered}
\varphi(\mathrm{z})= \\
{\left[_{\mathbf{n}=2}^{\Phi} \mathrm{P}^{\mathrm{X}}(\mathrm{e})(\ln \mathrm{p} \mathbf{n})(\mathrm{y})(\mathrm{i})\right]-1}
\end{gathered}
$$

So, it is not necessary that the branch of multivalued functions $\psi(z)$ and $\phi(z)$ have to be same all the time,In other words the value of $(e)^{\left(\ln p_{n}\right)(y)(i)}$ may be different for $\psi(z)$ and $\phi(z)$, If all

$$
\text { (e) } \frac{(\pi)(i)(\ln p)}{4 \ln 2}= \pm(i)
$$

and if $p_{n}$ denotes the $n$th prime then

$$
\begin{aligned}
\left(\sum_{n=1}^{\infty} \frac{1}{\left.(2 n-1)^{(} z_{1}\right)}\right) & =\prod_{n=2}^{\infty} \frac{1}{\left(1-\frac{1}{p_{n}^{Z_{1}}}\right)} \\
& =\prod_{n=2}^{\infty} \frac{1}{\left(1+\frac{ \pm i}{\sqrt{p_{n}}}\right)}
\end{aligned}
$$

and

$$
\begin{aligned}
\left(\sum_{n=1}^{\infty} \frac{1}{\left.(2 n-1)^{(} z_{2}\right)}\right) & =\prod_{n=2}^{\infty} \frac{1}{\left(1-\frac{1}{p_{n}^{Z_{2}}}\right)} \\
& =\prod_{n=2}^{\infty} \frac{1}{\left(1-\frac{ \pm i}{\sqrt{p_{n}}}\right)}
\end{aligned}
$$

Obviously then,

$$
\begin{aligned}
\left|\sum_{n=1}^{\infty} \frac{1}{\left.(2 n-1)^{(} z_{1}\right)}\right| & =\left|\sum_{n=1}^{\infty} \frac{1}{\left.(2 n-1)^{(} z_{2}\right)}\right| \\
& =\prod_{n=2}^{\infty} \frac{1}{\sqrt{\left(1+\frac{1}{p_{n}}\right)}}
\end{aligned}
$$

Since,

$$
\prod_{n=2}^{\infty} \frac{1}{\left(1+\frac{1}{p_{n}}\right)}
$$




$$
\begin{aligned}
& \times \prod_{n=2}^{\infty} \frac{1}{\left(1-\frac{1}{p_{n}}\right)} \\
& =\prod_{n=2}^{\infty} \frac{1}{\left(1-\frac{1}{p_{n}^{2}}\right)} \\
& =\sum_{n=1}^{\infty} \frac{1}{(2 n-1)^{2}} \\
& =\frac{(\pi)^{2}}{8}
\end{aligned}
$$

And

$$
\prod_{n=2}^{\infty} \frac{1}{\left(1-\frac{1}{p_{n}}\right)}=\frac{(\ln n)-\Upsilon}{2}
$$

Where $\Upsilon$ is EULER'S CONSTANT. Obviously then, from eqations $(1.3 .2,6),(1.3 .2 .7),(1.3 .2 .8),(1.3 .2 .11)$ and $(1.3 .2 .12)$ it follows,

$$
\begin{gathered}
\prod_{n=2}^{\infty} \frac{1}{\sqrt{\left(1+\frac{1}{p_{n}}\right)}}= \\
\frac{\pi}{2 \sqrt{(\ln n)-\Upsilon}}
\end{gathered}
$$

Consequently, when $n$ tends to $\infty$ then $\ln n \rightarrow \infty$ so $\frac{\pi}{2 \sqrt{(\ln n)-\Upsilon}} \rightarrow \sigma$, where $\sigma$ tends to zero. Therefore, from equations (1.3.2.6) and (1.3.2.13) it is clear that

$$
\begin{aligned}
\left|\sum_{n=1}^{\infty} \frac{1}{\left.(2 n-1)^{(} z_{1}\right)}\right| & =\left|\sum_{n=1}^{\infty} \frac{1}{\left.(2 n-1)^{(} z_{2}\right)}\right| \\
& =\sigma
\end{aligned}
$$

If all

$$
e^{\frac{(\ln p)(\pi)(i)}{4 \ln 2}}=-1
$$

then since,

$$
\begin{aligned}
& \left(\frac{1+\frac{1}{\sqrt{p}}}{\sqrt{\left(1+\frac{1}{P}\right)}}\right)^{2} \\
& 1+\frac{2 \sqrt{p}}{P+1}
\end{aligned}
$$

So, 


$$
\begin{aligned}
& \prod_{n=2}^{\infty} \frac{1}{\sqrt{\left(1+\frac{1}{P_{n}}\right)}}=\sigma> \\
& \prod_{n=2}^{\infty} \frac{1}{1+\frac{1}{\sqrt{P_{n}}}}
\end{aligned}
$$

$(\ln P)(\pi)(i)$

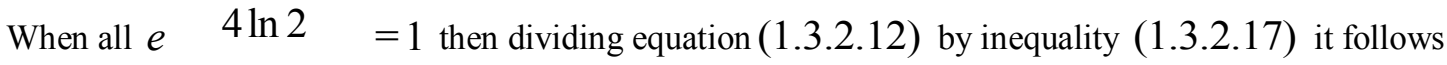

$$
\begin{aligned}
& \prod_{n=2}^{\infty} \frac{1}{1-\frac{1}{\sqrt{P_{n}}}}> \\
& \frac{(\ln n)-\Upsilon}{2}
\end{aligned}
$$

In otherwords, $\left|\sum_{n=1}^{\infty} \frac{1}{\left.(2 n-1)^{(} z_{1}\right)}\right|=\left|\sum_{n=1}^{\infty} \frac{1}{\left.(2 n-1)^{(} z_{2}\right)}\right| \rightarrow \infty$

$$
(\ln p)(\pi)(i)
$$

But if in this case,the value of $(e) \quad 4 \ln 2 \quad$ forthe function $\psi(z)$ be taken as +1 for the points $Z_{1}$ or $Z_{2}$ and -1 for the function $\phi(z)$, then it can be proved easily that $\left|\sum_{n=1}^{\infty} \frac{1}{\left.(2 n-1)^{(} z_{1}\right)}\right|=\left|\sum_{n=1}^{\infty} \frac{1}{\left.(2 n-1)^{(} z_{2}\right)}\right| \rightarrow \sigma$ where $\sigma$ tends to zero.In otherwords, the function $\sum_{n=1}^{\infty} \frac{1}{(2 n-1)^{Z}}$ is convergent on the real axis. So far we have considered those cases when all $e^{\frac{(\ln P)(\pi)(i)}{4 \ln 2}}$ is $\pm i$ or $(-1)$, but it may happens that for some finite number of primes(we denote any such primeby $p_{d}$ ) the value of $e^{\frac{\left(\ln p_{d}\right)(\pi)(i)}{4 \ln 2}}$ is different from the rest. But since $\prod_{d=d_{1}}^{d=d_{k}}\left(\frac{1}{1-\frac{1}{\left.\left(p_{d}\right)^{(} Z_{1}\right)}}\right)$ or $\prod_{d=d_{1}}^{d=d_{k}}\left(\frac{1}{1-\frac{1}{\left.\left(p_{d}\right)^{(} Z_{2}\right)}}\right) \quad$ is $\quad$ bounded $\quad$ so $\quad$ it $\quad$ can be proved easily that $\left|\sum_{n=1}^{\infty} \frac{1}{\left.(2 n-1)^{(} z_{1}\right)}\right|=\left|\sum_{n=1}^{\infty} \frac{1}{\left.(2 n-1)^{(} z_{2}\right)}\right| \rightarrow \sigma$ This completes proof of our assertion.

\subsection{3}

It is clear from our above discussion that if $Z=\frac{1}{t}+i \theta$, where $0<\left(\frac{1}{t}\right)<1$ then $\zeta(Z)$ is convergent as because

$$
\begin{aligned}
& |\zeta(Z)| \\
& \left(\sum_{n=1}^{\infty} \frac{1}{(2 n)^{Z}}|+| \sum_{n=1}^{\infty} \frac{1}{(2 n-1)^{Z}} \mid\right) \leq(m \sigma)
\end{aligned}
$$

where $m$ is some positive finite real number. Consequently, $\zeta(Z)$ is analytic on the right half plane i,e $R(z)>0$. We have already told that Since, 


$$
\left(\frac{1}{1-\frac{1}{P^{Z}}}\right)=\left(\frac{P^{Z}}{P^{Z}-1}\right)
$$

there is a possibility that in case of odd prime $P$, forany of $\left(\frac{P^{Z}}{P^{Z}-1}\right)$ the value of $\left(P^{Z}\right)$ of the numerator may be different from that of the denominator,as because $e^{(\ln p)(i)(\theta)}$ is infinitely many-valued. Let us explore such possibility for the point $z_{1}$ or $z_{2}$. Suppose,for any factor of $\sum_{n=1}^{\infty} \frac{1}{\left.(2 n-1)^{(} z_{1}\right)}$, for the $\left.P^{(} z_{1}\right)$ of the $\underline{(\ln p)(\pi)(i))}$

numerator, the value of $e^{4 \ln 2=-1}$ but for the $\left.P^{(} z_{1}\right)$ ofthe denominator, the value of $\underline{(\ln P)(\pi)(i)}$

$e^{4 \ln 2}=( \pm i)$ Then,

Consequently,

$$
\begin{aligned}
& \frac{\left.P^{(} z_{1}\right)}{\left.P^{(} z_{1}\right)-1}= \\
& \frac{ \pm i}{1+\frac{ \pm i}{\sqrt{P}}}
\end{aligned}=
$$

$$
\sum_{n=1}^{\infty} \frac{1}{\left.(2 n-1)^{(} z_{1}\right)}=( \pm i) \sum_{n=1}^{\infty} \frac{1}{\left.(2 n-1)^{(} z_{1}\right)}
$$

Consequently, When $\sum_{n=1}^{\infty} \frac{1}{\left.(2 n-1)^{(} z_{1}\right)}=-i \sum_{n=1}^{\infty} \frac{1}{\left.(2 n-1)^{(} z_{1}\right)}$, then for equation (1.2.1.2)

$$
\sum_{n=1}^{\infty} \frac{1}{\left.(2 n-1)^{(} z_{1}\right)}=-\sum_{n=1}^{\infty} \frac{1}{\left.(2 n)^{(} z_{1}\right)}
$$

Consequently,

$$
\zeta\left(z_{1}\right)=0
$$

When $\sum_{n=1}^{\infty} \frac{1}{\left.(2 n-1)^{(} z_{1}\right)}=+i \sum_{n=1}^{\infty} \frac{1}{\left.(2 n-1)^{(} z_{1}\right)}$, then for equation (1.2.1.2)

$$
\left(\sum_{n=1}^{\infty} \frac{1}{\left.(2 n-1)^{(} z_{1}\right)}-\sum_{n=1}^{\infty} \frac{1}{\left.(2 n)^{(} z_{1}\right)}\right)=0
$$

In otherwords, Dirichlet eta function becomes zero. Arguments are almost similar for $z_{2}$. On the otherhand,if none of the value of $\left.P^{(} z_{1}\right)$ or $\left.P^{(} z_{2}\right)$ of the numerator is different from that of the denominator,then for $z_{1}$, $\zeta\left(z_{1}\right) \neq 0 \quad$ but $\quad \sum_{n=1}^{\infty} \frac{1}{\left.(2 n-1)^{(} z_{1}\right)}-i \sum_{n=1}^{\infty} \frac{1}{\left.(2 n)^{(} z_{1}\right)}=0 \quad$ and $\quad$ for $\quad z_{2}, \quad \zeta\left(z_{2}\right) \neq 0 \quad$ but $\sum_{n=1}^{\infty} \frac{1}{\left.(2 n-1)^{(} z_{2}\right)}+i \sum_{n=1}^{\infty} \frac{1}{\left.(2 n)^{(} z_{2}\right)}=0$ Thus all non-trival roots of Riemann zeta function as well as roots of Dirichlet eta function lie on the line $Z=\frac{1}{2}+\left[\frac{(8 k \pm 1)(\pi)(i)}{4 \ln 2}\right]$ and this completes the proof of Riemann Hypothesis.

\section{References}

[1] KONRAD KNOPP.THEORY OF FUNCTIONS,5TH Edition.NEW YORK.DOVER PUBLICATIONS

[2] JOHN B.CONWAY.FUNCTIONS OF ONE COMPLEX VARIABLE,2nd Edition.NAROSA PUBLISHING HOUSE PVT LTD.

[3] J.N.SHARMA.FUNCTIONS OF A COMPLEX VARIABLE,4th Edition.Krishna Prakashan Media(P) Ltd. 